\title{
Stability of random implicit multifunctions in separable Asplund spaces
}

\author{
Ming-ge Yang ${ }^{\mathrm{a}, *}$, Yi-fan $\mathrm{Xu}$ \\ a School of Management, Shanghai University, Shanghai 200444, P. R. China. \\ ${ }^{b}$ School of Management, Fudan University, Shanghai 200433, P. R. China.
}

Communicated by Y. Z. Chen

\begin{abstract}
This paper is mainly devoted to present new sufficient conditions in terms of Fréchet coderivatives for the local metric regularity, the metric regularity, the Lipschitz-like property, the nonemptiness and the lower semicontinuity of random implicit multifunctions in separable Asplund spaces. An example is given to illustrate the above random implicit multifunction results. Some applications to stability analysis of solution maps for random parametric generalized equations are also given. (C)2017 All rights reserved.
\end{abstract}

Keywords: Fréchet coderivative, random implicit multifunction, (local) metric regularity, Lipschitz-like property, lower semicontinuity.

2010 MSC: 49J53, 90C31.

\section{Introduction}

Let $\mathrm{X}, \mathrm{P}$ be topological spaces, $\mathrm{Y}$ be a topological vector space, $\mathrm{F}: \mathrm{X} \times \mathrm{P} \rightrightarrows \mathrm{Y}$ be a multifunction, and $\left(x_{0}, p_{0}\right) \in X \times P$ be a pair such that

$$
0 \in \mathrm{F}\left(\mathrm{x}_{0}, \mathrm{p}_{0}\right) .
$$

The multifunction $\mathrm{G}: \mathrm{P} \rightrightarrows \mathrm{X}$ given by

$$
\mathrm{G}(\mathrm{p}):=\{x \in X \mid 0 \in \mathrm{F}(x, p)\},
$$

is called the implicit multifunction defined by the inclusion

$$
0 \in F(x, p) \text {. }
$$

The problem is to find some verifiable conditions on $F$ such that $G$ has the desirable properties. In the literature, different topological, metric and differential properties (e.g., lower semicontinuity, metric regularity, Lipschitz-like property, upper Lipschitz continuity, B-differentiability) of implicit multifunctions are considered. Of course the nonemptiness of the sets $G(p)$ for all $p$ in a neighborhood of $p_{0}$, is also an

\footnotetext{
${ }^{*}$ Corresponding author

Email addresses: mgyang@t.shu.edu.cn (Ming-ge Yang), yf xu@fudan.edu.cn (Yi-fan Xu)
} 
important property. The structure of $F$ and its behavior around $\left(x_{0}, p_{0}\right)$ decide local properties of $G$ in a neighborhood of the point $\left(p_{0}, x_{0}\right)$ in its graph.

The study of the stability of implicit multifunctions has a long history. The pioneering works of Robinson [12-15] gave good samples for implicit function theorems and their applications. Later, Ledyaev and Zhu [7], Ngai and Théra [11] established sufficient conditions for the metric regularity of the implicit multifunction (1.2) in terms of Fréchet coderivatives in Banach spaces with Fréchet-smooth Lipschitz bump functions. By using the scheme given by Yen [19], Lee et al. [8] showed some sufficient conditions for the nonemptiness, the lower semicontinuity, the metric regularity and the Lipschitz-like property of the implicit multifunction (1.2) in terms of normal coderivatives in Asplund spaces. Huy and Yao [6] established another set of sufficient conditions for the local metric regularity and the Lipschitz-like property of the implicit multifunction (1.2) in terms of normal coderivatives in Asplund spaces. Durea [2] obtained implicit multifunction results by simply specializing the openness results, and studied the metric regularity of implicit multifunctions in terms of normal coderivatives in Asplund spaces. Durea and Strugariu [3] also obtained implicit multifunction results by simply specializing the openness results, and studied the metric regularity and the Lipschitz-like property of implicit multifunctions in terms of Fréchet coderivatives in Asplund spaces. Huy et al. [5] gave new sufficient conditions for both the metric regularity and the Lipschitz-like property of implicit multifunctions in terms of Clarke coderivatives in general Banach spaces.

More recently, Yang and Huang [17] considered the following random implicit multifunction. Let $(\Omega, \mathcal{A})$ be a measurable space, $\mathrm{X}, \mathrm{P}$ be two topological spaces, $\mathrm{Y}$ be a topological vector space, $\mathrm{F}: \Omega \times \mathrm{X} \times$ $\mathrm{P} \rightrightarrows \mathrm{Y}$ be a multifunction, and $\left(\mathrm{x}_{0}, \mathrm{p}_{0}\right) \in \mathrm{X} \times \mathrm{P}$ be a pair such that

$$
0 \in F\left(\omega, x_{0}, p_{0}\right),
$$

for all $\omega \in \Omega$. The multifunction $\mathrm{G}: \Omega \times \mathrm{P} \rightrightarrows \mathrm{X}$ given by

$$
\mathrm{G}(\omega, \mathrm{p}):=\{x \in X \mid 0 \in \mathrm{F}(\omega, x, p)\},
$$

is called the random implicit multifunction defined by the inclusion

$$
0 \in \mathrm{F}(\omega, x, p),
$$

if for each $\mathrm{p} \in \mathrm{P}, \mathrm{G}(\cdot, \mathrm{p}): \Omega \rightrightarrows \mathrm{X}$ is measurable. By using normal coderivatives, Yang and Huang [17] gave sufficient conditions for the local metric regularity, the metric regularity, the Lipschitz-like property, the nonemptiness and the lower semicontinuity of the random implicit multifunction (1.3) in separable Asplund spaces. The results in [17] extended the corresponding results in [6, 8]. It is worth mentioning that implicit multifunction theorems in [17] are required the assumption on inner semicompactness of the metric projection mapping, while implicit multifunction theorems in $[2,3,5,18]$ are not required the assumption. So it is natural for us to discuss random implicit multifunction theorems without the assumption on inner semicompactness of the metric projection mapping in terms of Fréchet coderivatives.

The main purpose of this paper is to establish some random implicit multifunction theorems without the assumption on inner semicompactness of the metric projection mapping. Firstly, a random implicit multifunction theorem which gives sufficient conditions for the local metric regularity of the random implicit multifunction (1.3) is proved in separable Asplund spaces. Secondly, the metric regularity, the Lipschitz-like property, the nonemptiness and the lower semicontinuity of the random implicit multifunction (1.3) are discussed in separable Asplund spaces. An example is given to illustrate the above random implicit multifunction theorems. Finally, some applications to stability analysis for solution maps of random parametric generalized equations are given under suitable conditions. The results obtained in this paper are different from those in Yang and Huang [17], and generalize the corresponding results in $[2,3,5,6,8]$.

The paper is organized as follows. Section 2 recalls some basic concepts and results from variational analysis and generalized differentiation. Section 3 presents some random implicit multifunction theorems. Section 4 gives applications to stability analysis for solution maps of random parametric generalized equations. 


\section{Preliminaries}

Throughout this paper we use standard notation of variational analysis and generalized differentiation. We refer the reader to the books by Mordukhovich $[9,10]$ for more details and discussions. Unless otherwise stated, all spaces under consideration are Banach spaces whose norms are always denoted by $\|\cdot\|$. For any $X$, we consider its dual space $X^{*}$ equipped with the weak* topology $w^{*}$, where $\langle\cdot, \cdot\rangle$ means the canonical pairing. As usual, $\mathrm{B}_{X}$ and $\mathrm{B}_{\mathrm{X}^{*}}$ stand for the closed unit balls of the Banach space $\mathrm{X}$ and its dual $X^{*}$, respectively. The closed ball with center $x$ and radius $\rho$ is denoted by $B(x, \rho)$. The symbol $A^{*}$ is the adjoint operator of a linear continuous operator $A$. For a subset $\Omega \subset X, \operatorname{cl} \Omega$, int $\Omega, b d \Omega, \operatorname{co} \Omega$ and cone $\Omega$ denote, respectively, the closure, the interior, the boundary, the convex hull and the conic hull of $\Omega$. Throughout the paper, we use the notation

$$
x \stackrel{\Omega}{\rightarrow} \bar{x} \Longleftrightarrow x \rightarrow \bar{x} \text { with } x \in \Omega .
$$

Given a multifunction $\mathrm{F}: \mathrm{X} \rightrightarrows \mathrm{X}^{*}$ between a Banach space $\mathrm{X}$ and its topological dual $\mathrm{X}^{*}$, we denote by

$$
\text { Limsup }_{x \rightarrow \bar{x}} F(x):=\left\{x^{*} \in X^{*} \mid \exists \text { sequences } x_{k} \rightarrow \bar{x} \text { and } x_{k}^{*} \stackrel{w^{*}}{\longrightarrow} x^{*} \text { with } x_{k}^{*} \in F\left(x_{k}\right) \text { for all } k \in \mathbb{N}\right\},
$$

the sequential Painlevé-Kuratowski upper/outer limit with respect to the norm topology of $X$ and the weak* topology of $X^{*}$, where $\mathbb{N}:=\{1,2, \cdots\}$. Note that the symbol := means "equal by definition.

Definition 2.1 (generalized normals [9, Definition 1.1]). Let $\Omega$ be a nonempty subset of $X$.

(i) Given $x \in \Omega$ and $\varepsilon \geqslant 0$, define the set of $\varepsilon$-normals to $\Omega$ at $x$ by

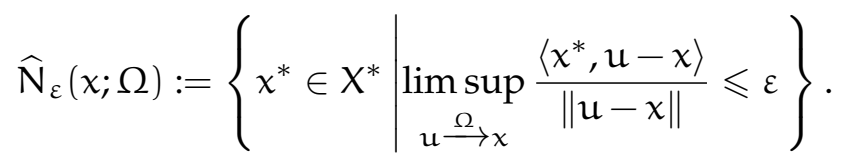

When $\varepsilon=0$, elements of (2.1) are called Fréchet normals and their collection, denoted by $\widehat{N}(x ; \Omega)$, is the prenormal cone to $\Omega$ at $x$. If $x \notin \Omega$, we put $\widehat{N}_{\varepsilon}(x ; \Omega):=\emptyset$ for all $\varepsilon \geqslant 0$.

(ii) Let $\bar{x} \in \Omega$. Then $x^{*} \in X^{*}$ is a basic/limiting normal to $\Omega$ at $\bar{x}$ if there are sequences $\varepsilon_{k} \downarrow 0, x_{k} \stackrel{\Omega}{\rightarrow} \bar{x}$ and $x_{k}^{*} \stackrel{w^{*}}{\longrightarrow} x^{*}$ such that $x_{k}^{*} \in \widehat{N}_{\varepsilon_{k}}\left(x_{k} ; \Omega\right)$ for all $k \in \mathbb{N}$. The collection of such normals

$$
\mathrm{N}(\overline{\mathrm{x}} ; \Omega):=\operatorname{Limsup}_{x \rightarrow \bar{x}, \mathcal{\varepsilon} \downarrow 0} \widehat{\mathrm{N}}_{\mathcal{\varepsilon}}(\mathrm{x} ; \Omega),
$$

is the (basic, limiting) normal cone to $\Omega$ at $\bar{x}$. Put $N(\bar{x} ; \Omega):=\emptyset$ if $\bar{x} \notin \Omega$.

A set $\Omega \subset X$ is sequentially normally compact (SNC) at $\bar{x} \in \Omega$ if for any sequence $\left(\varepsilon_{k}, x_{k}, x_{k}^{*}\right) \in$ $[0, \infty) \times \Omega \times X^{*}$ satisfying

$$
\varepsilon_{\mathrm{k}} \downarrow 0, x_{\mathrm{k}} \rightarrow \bar{x}, x_{\mathrm{k}}^{*} \in \widehat{\mathrm{N}}_{\varepsilon_{\mathrm{k}}}\left(x_{\mathrm{k}} ; \Omega\right) \text { and } x_{\mathrm{k}}^{*} \stackrel{w^{*}}{\longrightarrow} 0,
$$

one has $\left\|x_{k}^{*}\right\| \rightarrow 0$ as $k \rightarrow \infty$.

Let $\mathrm{F}: \mathrm{X} \rightrightarrows \mathrm{Y}$ a multifunction between topological spaces. Denote by

$$
\operatorname{dom} F:=\{x \in X \mid F(x) \neq \emptyset\}, \quad \operatorname{rgeF}:=\{y \in Y \mid \exists x \text { with } y \in F(x)\},
$$

the domain and the range of $F$. Each multifunction $F: X \rightrightarrows Y$ is uniquely associated with its graph

$$
\operatorname{gphF}:=\{(x, y) \in X \times Y \mid y \in F(x)\},
$$

in the product space $X \times Y$. The space $X \times Y$ is Banach with respect to the sum norm

$$
\|(x, y)\|:=\|x\|+\|y\|,
$$

imposed on $\mathrm{X} \times \mathrm{Y}$ unless otherwise stated. $\mathrm{F}$ is lower semicontinuous (in short, l.s.c.) at $\bar{\chi} \in$ domF if for 
any open set $V \subset Y$ satisfying $F(\bar{x}) \cap V \neq \emptyset$, there exists a neighborhood $U$ of $\bar{x}$ such that $F(x) \cap V \neq \emptyset$ for all $x \in U$. $F$ is lower/inner semicompact at $\bar{x} \in \operatorname{dom} F$ if for any sequence $x_{k} \rightarrow \bar{x}$, there is a sequence $y_{k} \in F\left(x_{k}\right)$ that contains a convergent subsequence as $k \rightarrow \infty$. It is clear that if $F$ is 1.s.c. at $\bar{x}$, then $F$ is inner semicompact at $\bar{x}$.

Definition 2.2 (coderivatives [9, Definition 1.32]). Let $F: X \rightrightarrows Y$ with $\operatorname{dom} F \neq \emptyset$.

(i) Given $(x, y) \in X \times Y$ and $\varepsilon \geqslant 0$, we define the $\varepsilon$-coderivative of $F$ at $(x, y)$ as a multifunction $\widehat{D}_{\varepsilon}^{*} \mathrm{~F}(x, y): Y^{*} \rightrightarrows X^{*}$ with the values

$$
\widehat{D}_{\varepsilon}^{*} \mathrm{~F}(x, y)\left(y^{*}\right):=\left\{x^{*} \in X^{*} \mid\left(x^{*},-y^{*}\right) \in \widehat{N}_{\varepsilon}((x, y) ; g p h F)\right\} \text {. }
$$

When $\varepsilon=0$, this construction is called the precoderivative or Fréchet coderivative of $F$ at $(x, y)$ and is denoted by $\widehat{D}^{*} F(x, y)$. It follows from the definition that $\widehat{D}_{\varepsilon}^{*} F(x, y)\left(y^{*}\right)=\emptyset$ for all $\varepsilon \geqslant 0$ and $y^{*} \in Y^{*}$ if $(x, y) \notin g p h F$.

(ii) Given $(x, y) \in X \times Y$, we define the normal coderivative of $F$ at $(x, y)$ as a multifunction $D_{N}^{*} F(x, y)$ : $Y^{*} \rightrightarrows X^{*}$ with the values

$$
\mathrm{D}_{\mathrm{N}}^{*} \mathrm{~F}(\mathrm{x}, \mathrm{y})\left(\mathrm{y}^{*}\right):=\left\{\mathrm{x}^{*} \in \mathrm{X}^{*} \mid\left(\mathrm{x}^{*},-\mathrm{y}^{*}\right) \in \mathrm{N}((\mathrm{x}, \mathrm{y}) ; \mathrm{gphF})\right\} .
$$

We put $D_{N}^{*} F(x, y)\left(y^{*}\right):=\emptyset$ for all $y^{*} \in Y^{*}$ if $(x, y) \notin g p h F$.

Recall that a single-valued mapping $\mathrm{f}: \mathrm{X} \rightarrow \mathrm{Y}$ is said to be Fréchet differentiable at $\bar{x}$ if there is a linear continuous operator $\nabla f(\bar{x}): X \rightarrow Y$, called the Fréchet derivative of $f$ at $\bar{x}$, such that

$$
\lim _{x \rightarrow \bar{x}} \frac{f(x)-f(\bar{x})-\nabla f(\bar{x})(x-\bar{x})}{\|x-\bar{x}\|}=0 .
$$

A mapping $f: X \rightarrow Y$ is said to be strictly differentiable at $\bar{x}$ if there is a linear continuous operator $\nabla f(\bar{x}): X \rightarrow Y$ such that

$$
\lim _{x, u \rightarrow \bar{x}} \frac{f(x)-f(u)-\nabla f(\bar{x})(x-u)}{\|x-u\|}=0 .
$$

Let $\varphi: X \rightarrow \overline{\mathbb{R}}$ be an extended real-valued function. We say that $\varphi$ is proper if $\varphi(x)>-\infty$ for all $x \in X$ and its domain

$$
\operatorname{dom} \varphi:=\{x \in X \mid \varphi(x)<\infty\},
$$

is nonempty. With any $\varphi$ we associate its epigraph

$$
\operatorname{epi} \varphi:=\{(x, \mu) \in X \times \mathbb{R} \mid \mu \geqslant \varphi(x)\} .
$$

Recall that $\varphi$ is 1.s.c. at a point $\bar{\chi}$ with $|\varphi(\bar{x})|<\infty$ if

$$
\liminf _{x \rightarrow \bar{x}} \varphi(x) \geqslant \varphi(\bar{x}) .
$$

We say that $\varphi$ is 1.s.c. around $\bar{\chi}$ when it is l.s.c. at any point of some neighborhood of $\bar{x}$.

Definition 2.3 (subdifferentials [9, Definition 1.77 and Definition 1.83]). Consider a function $\varphi: X \rightarrow \overline{\mathbb{R}}$ and a point $\bar{x} \in X$ with $|\varphi(\bar{x})|<\infty$.

(i) The set

$$
\widehat{\partial} \varphi(\bar{x})=\left\{x^{*} \in X^{*} \mid\left(x^{*},-1\right) \in \widehat{N}((\bar{x}, \varphi(\bar{x})) ; \operatorname{epi} \varphi)\right\},
$$

is the presubdifferential or Fréchet subdifferential of $\varphi$ at $\bar{x}$. We put $\widehat{\partial} \varphi(\bar{x}):=\emptyset$ if $|\varphi(\bar{x})|=\infty$. 
(ii) The set

$$
\partial \varphi(\bar{x}):=\left\{x^{*} \in X^{*} \mid\left(x^{*},-1\right) \in \mathrm{N}((\bar{x}, \varphi(\bar{x})) ; \operatorname{epi} \varphi)\right\},
$$

is the (basic, limiting) subdifferential of $\varphi$ at $\bar{x}$, and its elements are basic subgradients of $\varphi$ at this point. We put $\partial \varphi(\bar{x}):=\emptyset$ if $|\varphi(\bar{x})|=\infty$.

If $\varphi$ is convex, then both these subdifferentials do coincide with the classical Fenchel subdifferential. If $\delta_{\Omega}$ denotes the indicator function associated with a nonempty set $\Omega \subset$ X (i.e., $\delta_{\Omega}(x)=0$ if $x \in \Omega$, $\delta_{\Omega}(x)=+\infty$ if $\left.x \notin \Omega\right)$, then for any $\bar{x} \in \Omega, \widehat{\partial} \delta_{\Omega}(\bar{x})=\widehat{N}(\bar{x}, \Omega)$ and $\partial \delta_{\Omega}(\bar{x})=N(\bar{x}, \Omega)$.

Let $\varphi: X \rightarrow \overline{\mathbb{R}}$ be finite at $\bar{x}$. We say that $\varphi$ is sequentially normally epi-compact (SNEC) at $\bar{x}$ if its epigraph is SNC at $(\bar{x}, \varphi(\bar{x}))$. If $\varphi$ is Lipschitz continuous around $\bar{x}$, then it is SNEC at $\bar{x}$.

Lemma 2.4 (Ekeland's variational principle [9, Theorem 2.26]). Let (X, d) be a metric space. Assume that X is complete and that $\varphi: X \rightarrow \overline{\mathbb{R}}$ is a proper l.s.c. function bounded from below. Let $\varepsilon>0$ and $x_{0} \in X$ be given such that $\varphi\left(x_{0}\right) \leqslant \inf _{X} \varphi+\varepsilon$. Then for any $\lambda>0$ there is $\bar{x} \in X$ satisfying

(i) $\varphi(\bar{x}) \leqslant \varphi\left(x_{0}\right)$;

(ii) $\mathrm{d}\left(\overline{\mathrm{x}}, \mathrm{x}_{0}\right) \leqslant \lambda$;

(iii) $\varphi(x)+(\varepsilon / \lambda) d(x, \bar{x})>\varphi(\bar{x})$ for all $x \neq \bar{x}$.

Lemma 2.5 (nonsmooth versions of Fermat's rule [9, Proposition 1.114]). Let $\varphi: X \rightarrow \overline{\mathbb{R}}$ be finite at $\bar{x}$. Then

$$
0 \in \widehat{\partial} \varphi(\bar{x}) \subset \partial \varphi(\bar{x}),
$$

if $\varphi$ has a local minimum at $\bar{x}$.

A Banach space $X$ is Asplund, or it has the Asplund property, if every convex continuous function $\varphi: U \rightarrow \mathbb{R}$ defined on an open convex subset $U$ of $X$ is Fréchet differentiable on a dense subset of $U$; see [9, Definition 2.17]. The class of Asplund spaces is large. For instance, any reflexive Banach space is an Asplund space. The calculus of normal cones and subdifferentials in Asplund spaces is simpler than that in general Banach spaces; see [9, Chapter 3].

Lemma 2.6 (fuzzy sum rule [9, Theorem 2.33]). Let $\mathrm{X}$ be an Asplund space and $\varphi_{1}, \varphi_{2}: X \rightarrow \overline{\mathbb{R}}=\mathbb{R} \cup\{\infty\}$ be such that $\varphi_{1}$ is Lipschitz continuous around $\bar{x} \in \operatorname{dom} \varphi_{1} \cap \operatorname{dom} \varphi_{2}$ and $\varphi_{2}$ is l.s.c. around $\bar{x}$. Then for any $\gamma>0$ one has

$$
\widehat{\partial}\left(\varphi_{1}+\varphi_{2}\right)(\bar{x}) \subset \bigcup\left\{\widehat{\partial} \varphi_{1}\left(x_{1}\right)+\widehat{\partial} \varphi_{2}\left(x_{2}\right): x_{i} \in \bar{x}+\gamma B_{X},\left|\varphi_{i}\left(x_{i}\right)-\varphi_{i}(\bar{x})\right| \leqslant \gamma, i=1,2\right\}+\gamma B_{X^{*}} .
$$

Lemma 2.7 (coderivatives of differentiable mappings [9, Theorem 1.38]). Let $\mathrm{f}: \mathrm{X} \rightarrow \mathrm{Y}$ be Fréchet differentiable at $\bar{x}$. Then

$$
\widehat{D}^{*} f(\bar{x})\left(y^{*}\right)=\left\{\nabla f(\bar{x})^{*}\left(y^{*}\right)\right\}, \quad \forall y^{*} \in Y^{*}
$$

Lemma 2.8 (coderivative sum rules with equalities [9, Theorem 1.62]). Let $\mathrm{f}: \mathrm{X} \rightarrow \mathrm{Y}$ be Fréchet differentiable at $\bar{x}$, and let $\mathrm{F}: \mathrm{X} \rightrightarrows \mathrm{Y}$ be an arbitrary set-valued mapping such that $\overline{\mathrm{y}}-\mathrm{f}(\overline{\mathrm{x}}) \in \mathrm{F}(\overline{\mathrm{x}})$ for some $\overline{\mathrm{y}} \in \mathrm{Y}$. Then for all $\mathrm{y}^{*} \in \mathrm{Y}^{*}$, we have

$$
\widehat{D}^{*}(f+F)(\bar{x}, \bar{y})\left(y^{*}\right)=\nabla f(\bar{x})^{*} y^{*}+\widehat{D}^{*} F(\bar{x}, \bar{y}-f(\bar{x}))\left(y^{*}\right) .
$$

Consider the multifunction $G$ defined by (1.2) in a neighborhood of a point $\left(x_{0}, p_{0}\right)$ satisfying (1.1).

$\mathrm{G}$ is said to be locally metrically regular around $\left(x_{0}, p_{0}\right)$ with modulus $\gamma \geqslant 0$ if there are a neighborhood $V$ of $x_{0}$, a neighborhood $U$ of $p_{0}$, and a constant $\mu>0$ such that

$$
\operatorname{dist}(x, G(p)) \leqslant \gamma \operatorname{dist}(0, F(x, p)),
$$

for all $(x, p) \in \mathrm{V} \times \mathrm{U}$ with $\operatorname{dist}(0, \mathrm{~F}(x, p))<\mu$. The adverb "locally" will be omitted if the last inequality is not required for the fulfillment of (2.2). 
$\mathrm{G}$ is said to be Lipschitz-like around $\left(\mathrm{p}_{0}, \mathrm{x}_{0}\right)$ with modulus $l \geqslant 0$ if there are a neighborhood $\mathrm{U}$ of $\mathrm{p}_{0}$ and a neighborhood $V$ of $x_{0}$ such that

$$
\mathrm{G}\left(\mathrm{p}^{\prime}\right) \cap \mathrm{V} \subset \mathrm{G}(\mathrm{p})+\mathrm{l}\left\|\mathrm{p}^{\prime}-\mathrm{p}\right\| \mathrm{B}_{\mathrm{X}}, \quad \forall \mathrm{p}, \mathrm{p}^{\prime} \in \mathrm{U} .
$$

The Lipschitz-like property is also known as the pseudo-Lipschitzian property or the Aubin property of multifunctions.

At the end of this section, we give some basic concepts and results about measurability of multifunctions. For details, we refer the reader to Aubin and Frankowska [1, Chapter 8] and Himmelberg [4].

Consider a set $\Omega$ and a family $\mathcal{A}$ of subsets of $\Omega$. If $\mathcal{A}$ is a $\sigma$-algebra, we call the pair $(\Omega, \mathcal{A})$ a measurable space and the elements of $\mathcal{A}$ measurable sets. In case there is a $\sigma$-finite measure defined on $\mathcal{A}$, we say that $\Omega$ is $\sigma$-finite, and if there is a complete $\sigma$-finite measure defined on $\mathcal{A}$ we call $\Omega$ complete. Further, if $\Omega$ is a topological space, then the smallest $\sigma$-algebra containing all open sets is called the Borel $\sigma$-algebra. We denote it by $\mathcal{B}(\Omega)$ or simply $\mathcal{B}$, when it is clear from the context.

Definition 2.9 (measurability of multifunctions [4]). Consider a measurable space $(\Omega, \mathcal{A})$, a separable metrizable space $\mathrm{X}$, and a multifunction $\mathrm{F}: \Omega \rightrightarrows \mathrm{X}$. Then $\mathrm{F}$ is measurable (weakly measurable, $\mathcal{B}$ measurable, $\mathcal{C}$-measurable) if and only if

$$
\mathrm{F}^{-1}(\mathrm{~B}):=\{\omega \in \Omega: F(\omega) \cap B \neq \emptyset\}
$$

is measurable for each closed (resp., open, Borel, compact) subset $B$ of $X$.

If $F: Y \rightrightarrows X$ where $Y$ is a topological space, then the assertion that $F$ is measurable (weakly measurable, etc.) means that $F$ is measurable (weakly measurable, etc.) when $Y$ is assigned the $\sigma$-algebra $\mathcal{B}$ of Borel subsets of $\mathrm{Y}$. Likewise, if $\mathrm{F}: \Omega \times \mathrm{Y} \rightrightarrows \mathrm{X}$, then the various kinds of measurability of $\mathrm{F}$ are always defined in terms of the product $\sigma$-algebra $\mathcal{A} \times \mathcal{B}$ generated by the sets $\mathrm{A} \times \mathrm{B}$, where $\mathrm{A} \in \mathcal{A}$ and $\mathrm{B} \in \mathcal{B}$.

Lemma 2.10 (measurable projection [1, Theorem 8.3.2]). Let $(\Omega, \mathcal{A}, \mu)$ be a complete $\sigma$-finite measure space, $X$ a complete separable metric space and $\mathrm{G} \in \mathcal{A} \times \mathcal{B}(\mathrm{X})$. Then its projection is measurable:

$$
\pi_{\Omega}(\mathrm{G}):=\{\omega \in \Omega: \exists x \in X,(\omega, x) \in \mathrm{G}\} \in \mathcal{A} .
$$

\section{Random implicit multifunction theorems}

Theorem 3.1. Let $\mathrm{X}, \mathrm{Y}$ be separable Asplund spaces, $\mathrm{P}$ a topological space, $(\Omega, \mathcal{A}, \mu)$ a complete $\sigma$-finite measure space, $\mathrm{F}: \Omega \times \mathrm{X} \times \mathrm{P} \rightrightarrows \mathrm{Y}$ a multifunction such that for each $\mathrm{p} \in \mathrm{P}, \mathrm{F}(\cdot, \cdot, \mathrm{p}): \Omega \times \mathrm{X} \rightrightarrows \mathrm{Y}$ is measurable. Let $\mathrm{G}: \Omega \times \mathrm{P} \rightrightarrows \mathrm{X}$ be the multifunction defined by (1.3), and $\left(\mathrm{x}_{0}, \mathrm{p}_{0}\right) \in \mathrm{X} \times \mathrm{P}$ a pair such that $0 \in \mathrm{F}\left(\omega, \mathrm{x}_{0}, \mathrm{p}_{0}\right)$ for all $\omega \in \Omega$. Denote $\mathrm{F}_{\omega, \mathrm{p}}(\cdot):=\mathrm{F}(\omega, \cdot, \mathrm{p})$. Suppose that for each $\omega \in \Omega$, there exist constants $r>0$ and $\sigma>0$ such that:

(i) for any $\mathrm{p} \in \mathrm{B}\left(\mathrm{p}_{0}, \mathrm{r}\right)$, the multifunction $\mathrm{F}_{\mathrm{\omega}, \mathrm{p}}(\cdot)$ is closed;

(ii) for any $(x, p) \in \mathrm{B}\left(\mathrm{x}_{0}, \mathrm{r}\right) \times \mathrm{B}\left(\mathrm{p}_{0}, \mathrm{r}\right)$,

$$
\sigma \leqslant \inf \left\{\left\|x^{*}\right\|: x^{*} \in \widehat{D}^{*} F_{\omega, p}(x, y)\left(y^{*}\right), y \in B(0, r) \cap F_{\omega, p}(x),\left\|y^{*}\right\|=1\right\} .
$$

Then

(1) for any $\mathrm{p} \in \mathrm{P}, \mathrm{G}(\cdot, \mathrm{p}): \Omega \rightrightarrows \mathrm{X}$ is measurable;

(2) for any $\omega \in \Omega, \mathrm{G}(\omega, \cdot): \mathrm{P} \rightrightarrows \mathrm{X}$ is locally metrically regular around $\left(\mathrm{x}_{0}, \mathrm{p}_{0}\right)$ with modulus $\frac{1+\sigma}{\sigma}$. In fact, for any $\mu \in\left(0, \frac{r \sigma}{2(1+\sigma)}\right)$, we have

$$
\operatorname{dist}(x, \mathrm{G}(\omega, \mathrm{p})) \leqslant \frac{1+\sigma}{\sigma} \operatorname{dist}(0, \mathrm{~F}(\omega, x, \mathrm{p})),
$$

for all $(x, p) \in B\left(x_{0}, \frac{r}{2}\right) \times B\left(p_{0}, r\right)$ with $\operatorname{dist}(0, F(\omega, x, p))<\mu$. 
Proof. (1) Fix any $\mathrm{p} \in \mathrm{P}$, and consider the graph of $\mathrm{G}(\cdot, \mathrm{p}): \Omega \rightrightarrows \mathrm{X}$ which has the form

$$
\begin{aligned}
\operatorname{gphG}(\cdot, p) & =\{(\omega, x) \in \Omega \times X: x \in G(\omega, p)\} \\
& =\{(\omega, x) \in \Omega \times X: 0 \in F(\omega, x, p)\} \\
& =\{(\omega, x) \in \Omega \times X: F(\omega, x, p) \cap\{0\} \neq \emptyset\} .
\end{aligned}
$$

Since $F(\cdot, \cdot, p): \Omega \times X \rightrightarrows Y$ is measurable, we have that

$$
\{(\omega, x) \in \Omega \times X: F(\omega, x, p) \cap\{0\} \neq \emptyset\} \in \mathcal{A} \times \mathcal{B}(X),
$$

and it follows that

$$
\operatorname{gphG}(\cdot, \mathrm{p}) \in \mathcal{A} \times \mathcal{B}(X) .
$$

For any $B \in \mathcal{B}(X)$, by Lemma 2.10, we know that

$$
\pi_{\Omega}(\operatorname{gphG}(\cdot, \mathrm{p}) \cap(\Omega \times \mathrm{B})) \in \mathcal{A} .
$$

Since

$$
\begin{aligned}
\pi_{\Omega}(\operatorname{gph}(\cdot, p) \cap(\Omega \times B)) & =\{\omega \in \Omega: \exists x \in X,(\omega, x) \in \operatorname{gphG}(\cdot, p) \cap(\Omega \times B)\} \\
& =\{\omega \in \Omega: \exists x \in B, x \in G(\omega, p)\} \\
& =\{\omega \in \Omega: G(\omega, p) \cap B \neq \emptyset\},
\end{aligned}
$$

we obtain that

$$
\{\omega \in \Omega: G(\omega, p) \cap B \neq \emptyset\} \in \mathcal{A} .
$$

Hence, $\mathrm{G}(\cdot, \mathrm{p}): \Omega \rightrightarrows X$ is $\mathcal{B}$-measurable. It follows that $\mathrm{G}(\cdot, \mathrm{p}): \Omega \rightrightarrows \mathrm{X}$ is measurable.

(2) Fix any $\omega \in \Omega$. By the assumptions, there exist constants $r>0$ and $\sigma>0$ such that conditions (i) and (ii) are satisfied. Fix any $\mu \in\left(0, \frac{r \sigma}{2(1+\sigma)}\right)$, and any $(x, p) \in B\left(x_{0}, \frac{r}{2}\right) \times B\left(p_{0}, r\right)$ with $\operatorname{dist}(0, F(\omega, x, p))<\mu$. Now we prove that (3.1) holds. If $\operatorname{dist}(0, F(\omega, x, p))=0$, then $0 \in F(\omega, x, p)$ and hence $x \in G(\omega, p)$. Therefore, both sides of (3.1) are equal to 0 and (3.1) holds. Hence, we can assume that dist $(0, F(\omega, x, p))=$ $\alpha$, where $\alpha \in(0, \mu)$. It remains to show that

$$
\operatorname{dist}(x, G(\omega, p)) \leqslant \frac{\alpha(1+\sigma)}{\sigma} .
$$

Since $0<\alpha<\mu<\frac{r \sigma}{2(1+\sigma)}$, we obtain that $\frac{2 \alpha}{r}<\frac{\sigma}{1+\sigma}$. For each $\varepsilon \in(0, r-\mu)$ with $\frac{2(\alpha+\varepsilon)}{r}<\frac{\sigma}{1+\sigma}$, by the definition of the distance function, there exists $\bar{y} \in F_{\omega, p}(x)$ such that $\|\bar{y}\|<\alpha+\varepsilon<\mu+\varepsilon<r$. Define the function $f_{p}: X \times Y \rightarrow \overline{\mathbb{R}}$ by

$$
f_{p}\left(x^{\prime}, y^{\prime}\right):=\left\|y^{\prime}\right\|+\delta\left(\left(x^{\prime}, y^{\prime}\right) ; g p h F_{w, p}\right), \quad \forall\left(x^{\prime}, y^{\prime}\right) \in X \times Y .
$$

We claim that $f_{p}$ is 1.s.c. on $X \times Y$ due to condition (i). Fix any $t \in\left(\frac{2(\alpha+\varepsilon)}{r}, \frac{\sigma}{1+\sigma}\right)$, and put $\beta:=f_{p}(x, \bar{y})=$ $\|\bar{y}\|$. We see that

$$
f_{p}(x, \bar{y})=t \cdot \frac{\beta}{t}
$$

Clearly,

$$
f_{p}(x, \bar{y}) \leqslant \inf _{\left(x^{\prime}, y^{\prime}\right) \in B\left(x_{0}, r\right) \times B(0, r)} f_{p}\left(x^{\prime}, y^{\prime}\right)+t \cdot \frac{\beta}{t} .
$$

By the Ekeland variational principle in Lemma 2.4, there exists $(\hat{x}, \hat{y}) \in B\left(x_{0}, r\right) \times B(0, r)$ such that

$$
f_{p}(\hat{x}, \hat{y}) \leqslant f_{p}(x, \bar{y}), \quad\|(\hat{x}, \hat{y})-(x, \bar{y})\| \leqslant \frac{\beta}{t}
$$


and

$$
f_{p}(\hat{x}, \hat{y}) \leqslant f_{p}\left(x^{\prime}, y^{\prime}\right)+t \cdot\left\|\left(x^{\prime}, y^{\prime}\right)-(\hat{x}, \hat{y})\right\|, \quad \forall\left(x^{\prime}, y^{\prime}\right) \in B\left(x_{0}, r\right) \times B(0, r) .
$$

This implies that $(\hat{x}, \hat{y}) \in g_{p h F}$,

$$
\|\hat{y}\| \leqslant\|\bar{y}\|, \quad\|\hat{x}-x\|+\|\hat{y}-\bar{y}\| \leqslant \frac{\beta}{t},
$$

and

$$
\|\hat{y}\| \leqslant\left\|y^{\prime}\right\|+\mathfrak{t}\left(\left\|x^{\prime}-\hat{x}\right\|+\left\|y^{\prime}-\hat{y}\right\|\right)+\delta\left(\left(x^{\prime}, y^{\prime}\right) ; \operatorname{gphF}_{w, p}\right), \quad \forall\left(x^{\prime}, y^{\prime}\right) \in B\left(x_{0}, r\right) \times B(0, r) .
$$

We now show that $0 \in F_{\omega, p}(\hat{x})$. Assume to the contrary that $0 \notin F_{\omega, p}(\hat{x})$, then $\hat{y} \neq 0$. Obviously, $\hat{x} \in B\left(x_{0}, r\right), \hat{y} \in B(0, r)$. Since

$$
\left\|\hat{x}-x_{0}\right\| \leqslant\|\hat{x}-x\|+\left\|x-x_{0}\right\| \leqslant \frac{\beta}{t}+\frac{r}{2}<\frac{\alpha+\varepsilon}{t}+\frac{r}{2}<\frac{r}{2}+\frac{r}{2}=r,
$$

and

$$
\|\hat{y}\| \leqslant\|\bar{y}\|=\beta<\alpha+\varepsilon<\mu+\varepsilon<r,
$$

we have that $(\hat{x}, \hat{y}) \in \operatorname{int} B\left(x_{0}, r\right) \times \operatorname{int} B(0, r)=\operatorname{int}\left(B\left(x_{0}, r\right) \times B(0, r)\right)$. Define functions $\varphi_{1}, \varphi_{2}, \varphi_{3}: X \times Y \rightarrow$ $\overline{\mathbb{R}}$ by

$$
\begin{aligned}
& \varphi_{1}\left(x^{\prime}, y^{\prime}\right):=\left\|y^{\prime}\right\|, \\
& \varphi_{2}\left(x^{\prime}, y^{\prime}\right):=t\left(\left\|x^{\prime}-\hat{x}\right\|+\left\|y^{\prime}-\hat{y}\right\|\right), \\
& \varphi_{3}\left(x^{\prime}, y^{\prime}\right):=\delta\left(\left(x^{\prime}, y^{\prime}\right) ; g p h F_{w, p}\right),
\end{aligned}
$$

for all $\left(x^{\prime}, y^{\prime}\right) \in X \times Y$.

It follows from (3.2) that $(\hat{x}, \hat{y})$ is a local minimum of the function $\varphi_{1}+\varphi_{2}+\varphi_{3}$ on $X \times Y$. From the nonsmooth version of Fermat's rule in Lemma 2.5, we know that

$$
(0,0) \in \widehat{\partial}\left(\varphi_{1}+\varphi_{2}+\varphi_{3}\right)(\hat{x}, \hat{y}) .
$$

Using the fact that $\varphi_{1}+\varphi_{2}$ is Lipschitz continuous on $X \times Y$ and $\varphi_{3}$ is 1.s.c on $X \times Y$, we can apply the fuzzy sum rule for the Fréchet subdifferential. Choose $0<\gamma<\min \left\{1-t, \frac{\sigma-(1+\sigma) t}{1+\sigma}\right\}$ with $0 \notin B(\hat{y}, \gamma)$, $\mathrm{B}(\hat{x}, \gamma) \subset \mathrm{B}\left(\mathrm{x}_{0}, \mathrm{r}\right)$ and $\mathrm{B}(\hat{\mathrm{y}}, \gamma) \subset \mathrm{B}(0, r)$. By Lemma 2.6, there exist

$$
\left(x_{\gamma}^{1}, y_{\gamma}^{1}\right) \in B(\hat{x}, \gamma) \times B(\hat{y}, \gamma) \subset B\left(x_{0}, r\right) \times B(0, r),
$$

and

such that

$$
\left(x_{\gamma}^{2}, y_{\gamma}^{2}\right) \in[B(\hat{x}, \gamma) \times B(\hat{y}, \gamma)] \cap g_{p h} F_{\omega, p} \subset\left[B\left(x_{0}, r\right) \times B(0, r)\right] \cap g p h F_{\omega, p},
$$

$$
(0,0) \in \widehat{\partial}\left(\varphi_{1}+\varphi_{2}\right)\left(x_{\gamma}^{1}, y_{\gamma}^{1}\right)+\widehat{\partial} \varphi_{3}\left(x_{\gamma}^{2}, y_{\gamma}^{2}\right)+\gamma\left(B_{X^{*}} \times B_{Y^{*}}\right)
$$

Morever,

$$
\left|\left(\varphi_{1}+\varphi_{2}\right)\left(x_{\gamma}^{1}, y_{\gamma}^{1}\right)-\left(\varphi_{1}+\varphi_{2}\right)(\hat{x}, \hat{y})\right| \leqslant \gamma, \quad \text { and }\left|\varphi_{3}\left(x_{\gamma}^{2}, y_{\gamma}^{2}\right)-\varphi_{3}(\hat{x}, \hat{y})\right| \leqslant \gamma
$$

Observing that $\varphi_{1}$ and $\varphi_{2}$ are convex functions and $y_{\gamma}^{1} \neq 0$, we obtain that

$$
(0,0) \in\{0\} \times \mathrm{S}_{Y^{*}}+\mathrm{t}\left(\mathrm{B}_{\mathbf{X}^{*}} \times\{0\}+\{0\} \times \mathrm{B}_{\mathrm{Y}^{*}}\right)+\widehat{\mathrm{N}}\left(\left(x_{\gamma^{\prime}}^{2}, \mathrm{y}_{\gamma}^{2}\right) ; \operatorname{gph}_{\omega, p}\right)+\gamma\left(\mathrm{B}_{\mathbf{X}^{*}} \times \mathrm{B}_{\mathrm{Y}^{*}}\right) .
$$

Hence, there exist $y_{1}^{*} \in S_{Y^{*}},\left(x_{2}^{*}, y_{2}^{*}\right) \in \widehat{N}\left(\left(x_{\gamma}^{2}, y_{\gamma}^{2}\right) ; g p h F_{\omega, p}\right), x_{3}^{*} \in B_{X^{*}}$ and $y_{3}^{*} \in B_{Y^{*}}$ such that

$$
\left\|y_{1}^{*}\right\|=1, \quad\left\|x_{2}^{*}+\gamma x_{3}^{*}\right\| \leqslant t, \quad \text { and }\left\|y_{1}^{*}+y_{2}^{*}+\gamma y_{3}^{*}\right\| \leqslant t .
$$

Let $x^{*}:=\frac{x_{2}^{*}}{\left\|y_{2}^{*}\right\|}$ and $y^{*}:=-\frac{y_{2}^{*}}{\left\|y_{2}^{*}\right\|}$. Then we have

$$
\left(x^{*},-y^{*}\right) \in \widehat{N}\left(\left(x_{\gamma}^{2}, y_{\gamma}^{2}\right) ; g p h F_{\omega, p}\right) .
$$


It follows that

$$
x^{*} \in \widehat{D}^{*} F_{\omega, p}\left(x_{\gamma}^{2}, y_{\gamma}^{2}\right)\left(y^{*}\right)
$$

Since $\left\|x_{2}^{*}\right\|-\left\|\gamma x_{3}^{*}\right\| \leqslant\left\|x_{2}^{*}+\gamma x_{3}^{*}\right\| \leqslant t$, we obtain that $\left\|x_{2}^{*}\right\| \leqslant \gamma+t$. Furthermore,

$$
t \geqslant\left\|y_{1}^{*}+y_{2}^{*}+\gamma y_{3}^{*}\right\| \geqslant\left\|y_{1}^{*}\right\|-\left\|y_{2}^{*}+\gamma y_{3}^{*}\right\| \geqslant 1-\left\|y_{2}^{*}\right\|-\gamma\left\|y_{3}^{*}\right\| \geqslant 1-\left\|y_{2}^{*}\right\|-\gamma
$$

Hence, $\left\|y_{2}^{*}\right\| \geqslant 1-\gamma-t$. It follows that

$$
\left\|x^{*}\right\|=\frac{\left\|x_{2}^{*}\right\|}{\left\|y_{2}^{*}\right\|} \leqslant \frac{\gamma+t}{1-\gamma-t}<\sigma,
$$

which is contrary to condition (ii). Therefore, we have shown that $0 \in F_{\omega, p}(\hat{x})$, i.e. $\hat{x} \in G(\omega, p)$. It follows that

$$
\operatorname{dist}(x, G(\omega, p)) \leqslant\|x-\hat{x}\| \leqslant \frac{\beta}{t}<\frac{\alpha+\varepsilon}{t} .
$$

Since $t<\frac{\sigma}{1+\sigma}$, by letting $t \rightarrow \frac{\sigma}{1+\sigma}$ and $\varepsilon \rightarrow 0$, we have that

$$
\operatorname{dist}(x, G(\omega, p)) \leqslant \frac{\alpha(1+\sigma)}{\sigma}=\frac{1+\sigma}{\sigma} \operatorname{dist}(0, F(\omega, x, p))
$$

Remark 3.2. We notice that [17, Theorem 3.1] is required the assumption on inner semicompactness of the metric projection mapping, but Theorem 3.1 is not required. Moreover, [17, Theorem 3.1] uses normal coderivatives, while Theorem 3.1 uses Fréchet coderivatives. Also, we can see from the proof of Theorem 3.1 that the conclusion of the theorem is still valid, if the topological space $\mathrm{P}$ is replaced by a metric space.

When considering the deterministic case of Theorem 3.1, we have the following corollary.

Corollary 3.3. Let $\mathrm{X}, \mathrm{Y}$ be Asplund spaces, $\mathrm{P}$ a topological space, $\mathrm{F}: \mathrm{X} \times \mathrm{P} \rightrightarrows \mathrm{Y}$ a multifunction, $\mathrm{G}: \mathrm{P} \rightrightarrows \mathrm{X}$ the implicit multifunction defined by (1.2), and $\left(\mathrm{x}_{0}, \mathrm{p}_{0}\right) \in \mathrm{X} \times \mathrm{P}$ a pair such that $0 \in \mathrm{F}\left(\mathrm{x}_{0}, \mathrm{p}_{0}\right)$. Denote $\mathrm{F}_{\mathrm{p}}(\cdot):=\mathrm{F}(\cdot, \mathrm{p})$. Suppose that there exist constants $r>0$ and $\sigma>0$ such that:

(i) for any $\mathrm{p} \in \mathrm{B}\left(\mathrm{p}_{0}, \mathrm{r}\right)$, the multifunction $\mathrm{F}_{\mathrm{p}}(\cdot)$ is closed;

(ii) for any $(x, p) \in B\left(x_{0}, r\right) \times B\left(p_{0}, r\right)$,

$$
\sigma \leqslant \inf \left\{\left\|x^{*}\right\|: x^{*} \in \widehat{D}^{*} F_{p}(x, y)\left(y^{*}\right), y \in B(0, r) \cap F_{p}(x),\left\|y^{*}\right\|=1\right\}
$$

Then $\mathrm{G}$ is locally metrically regular around $\left(\mathrm{x}_{0}, \mathrm{p}_{0}\right)$ with modulus $\frac{1+\sigma}{\sigma}$. In fact, for any $\mu \in\left(0, \frac{\mathrm{r} \sigma}{2(1+\sigma)}\right)$, we have

$$
\operatorname{dist}(x, \mathrm{G}(\mathrm{p})) \leqslant \frac{1+\sigma}{\sigma} \operatorname{dist}(0, \mathrm{~F}(\mathrm{x}, \mathrm{p})),
$$

for all $(x, \mathrm{p}) \in \mathrm{B}\left(\mathrm{x}_{0}, \frac{\mathrm{r}}{2}\right) \times \mathrm{B}\left(\mathrm{p}_{0}, \mathrm{r}\right)$ with $\operatorname{dist}(0, \mathrm{~F}(\mathrm{x}, \mathrm{p}))<\mu$.

Remark 3.4. We notice that [6, Theorem 3.5] is required the assumption on inner semicompactness of the projection mapping, but Corollary 3.3 is not required. Corollary 3.3 is also similar with [5, Theorem 3.1], which is based on Clarke coderivative and Clarke subdifferential in Banach spaces. By using different norm on the product space, the proof of Corollary 3.3 is much simpler than that of [5, Theorem 3.1].

Theorem 3.5. Suppose that all the assumptions of Theorem 3.1 are satisfied. Moreover, assume that

(iii) $)_{1}$ for any $\omega \in \Omega, \mathrm{F}(\omega, \cdot, \cdot)$ is l.s.c. at $\left(\mathrm{x}_{0}, \mathrm{p}_{0}\right)$.

Then

(1) for any $\mathrm{p} \in \mathrm{P}, \mathrm{G}(\cdot, \mathrm{p}): \Omega \rightrightarrows \mathrm{X}$ is measurable; 
(2) for any $\omega \in \Omega, \mathrm{G}(\omega, \cdot): \mathrm{P} \rightrightarrows \mathrm{X}$ is metrically regular around $\left(\mathrm{x}_{0}, \mathrm{p}_{0}\right)$ with modulus $\frac{1+\sigma}{\sigma}$. In fact, there exist a neighborhood $\mathrm{V}$ of $\mathrm{x}_{0}$ and a neighborhood $\mathrm{U}$ of $\mathrm{p}_{0}$ such that

$$
\operatorname{dist}(x, \mathrm{G}(\omega, \mathrm{p})) \leqslant \frac{1+\sigma}{\sigma} \operatorname{dist}(0, \mathrm{~F}(\omega, x, \mathrm{p})),
$$

for all $(x, \mathrm{p}) \in \mathrm{V} \times \mathrm{U}$.

Remark 3.6. Since the proof of Theorem 3.5 is similar to that of [17, Theorem 3.5], we omit the proof here. It is worth mentioning that Theorem 3.5 is different from [17, Theorem 3.5], because the assumptions in Theorem 3.5 is different from that in [17, Theorem 3.5].

Theorem 3.7. Suppose that all the assumptions of Theorem 3.1 are satisfied. Moreover, assume that $\mathrm{P}$ is a subset of a normed space and

(iii) $)_{2}$ for any $\omega \in \Omega$, there exist a neighborhood $\mathrm{V}$ of $\mathrm{x}_{0}$, a neighborhood $\mathrm{U}$ of $\mathrm{p}_{0}$ and a constant $\mathrm{l}>0$ such that

$$
\mathrm{F}\left(\omega, x, p^{\prime}\right) \subset \mathrm{F}(\omega, x, p)+l\left\|p^{\prime}-p\right\| B_{Y}, \quad \forall x \in V, \quad \forall p, p^{\prime} \in \mathrm{U} .
$$

Then

(1) for any $\mathrm{p} \in \mathrm{P}, \mathrm{G}(\cdot, \mathrm{p}): \Omega \rightrightarrows \mathrm{X}$ is measurable;

(2) for any $\omega \in \Omega, \mathrm{G}(\omega, \cdot): \mathrm{P} \rightrightarrows \mathrm{X}$ is Lipschitz-like around $\left(\mathrm{p}_{0}, \mathrm{x}_{0}\right)$.

Remark 3.8. Since the proof of Theorem 3.7 is similar to that of [17, Theorem 3.8], we omit the proof here. It is worth mentioning that Theorem 3.7 is different from [17, Theorem 3.8], because the assumptions in Theorem 3.7 is different from that in [17, Theorem 3.8].

Theorem 3.9. Suppose that all the assumptions of Theorem 3.1 are satisfied. Moreover, assume that

(iii) $)_{3}$ for any $(\omega, x, p) \in \Omega \times \mathrm{B}\left(\mathrm{x}_{0}, \mathrm{r}\right) \times \mathrm{B}\left(\mathrm{p}_{0}, \mathrm{r}\right)$, the multifunction $\mathrm{F}(\omega, \mathrm{x}, \cdot)$ is l.s.c. at $\mathrm{p}$.

Then

(1) for any $\mathrm{p} \in \mathrm{P}, \mathrm{G}(\cdot, \mathrm{p}): \Omega \rightrightarrows \mathrm{X}$ is measurable;

(2) for any $\omega \in \Omega$, there exists a constant $\mathrm{s} \in(0, \mathrm{r})$ such that the multifunction $\widetilde{\mathrm{G}}_{\omega}: \mathrm{P} \rightrightarrows \mathrm{X}$ defined by

$$
\widetilde{\mathrm{G}}_{\omega}(\mathrm{p}):=\mathrm{G}(\omega, \mathrm{p}) \cap \operatorname{int} \mathrm{B}\left(\mathrm{x}_{0}, \mathrm{r}\right),
$$

is nonempty and l.s.c. on $\mathrm{B}\left(\mathrm{p}_{0}, \mathrm{~s}\right)$.

Proof. By Theorem 3.1, conclusion (1) of Theorem 3.9 holds. Now we show that conclusion (2) of Theorem 3.9 also holds. Fix any $\omega \in \Omega$. Since $0 \in F\left(\omega, x_{0}, p_{0}\right)$, by condition (iii) $)_{3}$, there exists a neighborhood $\widetilde{U}$ of $p_{0}$ such that

$$
\mathrm{F}\left(\omega, x_{0}, p\right) \cap \operatorname{intB}\left(0, \frac{r \sigma}{1+\sigma}\right) \neq \emptyset,
$$

for every $p \in \widetilde{\mathrm{U}}$. Hence,

$$
\operatorname{dist}\left(0, F\left(\omega, x_{0}, p\right)\right)<\frac{r \sigma}{1+\sigma}
$$

for every $p \in \widetilde{U}$. Choose a number $s \in(0, r)$ satisfying $B\left(p_{0}, s\right) \subset \widetilde{U}$. We want to show that $s$ satisfies the conclusion (2) of Theorem 3.9.

(a) Fix any $p \in B\left(p_{0}, s\right)$. Now we show that $\widetilde{G}_{\omega}(p)$ is nonempty. Define the function $f_{p}: X \times Y \rightarrow \overline{\mathbb{R}}$ by

$$
f_{p}(x, y):=\|y\|+\delta\left((x, y) ; \operatorname{gphF}_{w, p}\right), \quad \forall(x, y) \in X \times Y .
$$

We claim that $f_{p}$ is 1.s.c. on $X \times Y$ due to condition (i). In particular, it is 1.s.c. on $B\left(x_{0}, r\right) \times B(0, r)$. If 
$f_{p}\left(x_{0}, 0\right)=0$, then $0 \in F_{\omega, p}\left(x_{0}\right)$, and hence $x_{0} \in G(\omega, p)$. It follows that $x_{0} \in G(\omega, p) \cap \operatorname{int} B\left(x_{0}, r\right)$. That is $\widetilde{\mathrm{G}}_{\omega}(\mathrm{p}) \neq \emptyset$. If $f_{p}\left(x_{0}, 0\right) \neq 0$, then $0 \notin \mathrm{F}_{\omega, p}\left(x_{0}\right)$, and hence $\operatorname{dist}\left(0, F\left(\omega, x_{0}, p\right)\right)>0$. We can assume that $\alpha:=\operatorname{dist}\left(0, F\left(\omega, x_{0}, p\right)\right)$, where $0<\alpha<\frac{r \sigma}{1+\sigma}<r$.

For each $\varepsilon \in(0, r-\alpha)$ with $\frac{\alpha+\varepsilon}{r}<\frac{\sigma}{1+\sigma}$, by the definition of the distance function, there exists $\bar{y} \in$ $F_{\omega, p}\left(x_{0}\right)$ such that $\|\bar{y}\|<\alpha+\varepsilon<r$. Let $\beta:=f_{p}\left(x_{0}, \bar{y}\right)=\|\bar{y}\|$, and fix any $t \in\left(\frac{\alpha+\varepsilon}{r}, \frac{\sigma}{1+\sigma}\right)$. We see that

$$
f_{p}\left(x_{0}, \bar{y}\right)=t \cdot \frac{\beta}{t}
$$

Clearly,

$$
f_{p}\left(x_{0}, \bar{y}\right) \leqslant \inf _{(x, y) \in B\left(x_{0}, r\right) \times B(0, r)} f_{p}(x, y)+t \cdot \frac{\beta}{t} .
$$

By the Ekeland variational principle in Lemma 2.4 , there exists $(\hat{x}, \hat{y}) \in B\left(x_{0}, r\right) \times B(0, r)$ such that

$$
f_{p}(\hat{x}, \hat{y}) \leqslant f_{p}\left(x_{0}, \bar{y}\right), \quad\left\|(\hat{x}, \hat{y})-\left(x_{0}, \bar{y}\right)\right\| \leqslant \frac{\beta}{t}
$$

and

$$
f_{p}(\hat{x}, \hat{y}) \leqslant f_{p}(x, y)+t \cdot\|(x, y)-(\hat{x}, \hat{y})\|, \quad \forall(x, y) \in B\left(x_{0}, r\right) \times B(0, r) .
$$

This implies that $(\hat{x}, \hat{y}) \in \operatorname{gphF}_{w, p}$,

$$
\|\hat{y}\| \leqslant\|\bar{y}\|, \quad\left\|\hat{x}-x_{0}\right\|+\|\hat{y}-\bar{y}\| \leqslant \frac{\beta}{t},
$$

and

$$
\|\hat{y}\| \leqslant\|y\|+t(\|x-\hat{x}\|+\|y-\hat{y}\|)+\delta\left((x, y) ; g p h F_{w, p}\right), \quad \forall(x, y) \in B\left(x_{0}, r\right) \times B(0, r) .
$$

Obviously, $\hat{x} \in B\left(x_{0}, r\right), \hat{y} \in B(0, r)$. Since

$$
\left\|\hat{x}-x_{0}\right\| \leqslant \frac{\beta}{t}<\frac{\alpha+\varepsilon}{t}<r, \quad\|\hat{y}\| \leqslant\|\bar{y}\|<r,
$$

we have that $(\hat{x}, \hat{y}) \in \operatorname{int} B\left(x_{0}, r\right) \times \operatorname{int} B(0, r)=\operatorname{int}\left(B\left(x_{0}, r\right) \times B(0, r)\right)$.

We now show that $0 \in F_{\omega, p}(\hat{x})$. Assume to the contrary that $0 \notin F_{\omega, p}(\hat{x})$, then $\hat{y} \neq 0$. Define functions $\varphi_{1}, \varphi_{2}, \varphi_{3}: X \times Y \rightarrow \overline{\mathbb{R}}$ by

$$
\varphi_{1}(x, y):=\|y\|, \varphi_{2}(x, y):=t(\|x-\hat{x}\|+\|y-\hat{y}\|), \varphi_{3}(x, y):=\delta\left((x, y) ; g p h F_{w, p}\right), \quad \forall(x, y) \in X \times Y .
$$

It follows from (3.3) that $(\hat{x}, \hat{y})$ is a local minimum of the function $\varphi_{1}+\varphi_{2}+\varphi_{3}$ on $X \times Y$. Arguing as in Theorem 3.1, we can deduce a contradiction with condition (ii). Therefore, we have shown that $0 \in \mathrm{F}_{\omega, \mathrm{p}}(\hat{x})$, i.e., $\hat{x} \in \mathrm{G}(\omega, \mathrm{p})$. It follows that $\widetilde{\mathrm{G}}_{\omega}(\mathrm{p}) \neq \emptyset$.

(b) Fix any $p \in B\left(p_{0}, s\right)$, and we show that $\widetilde{G}_{\omega}(\cdot)$ is l.s.c. at $p$. It suffices to show that for any $x \in \widetilde{G}_{\omega}(p)$ and any $\varepsilon>0$, there exists a constant $t>0$ such that

$$
\widetilde{\mathrm{G}}_{\omega}\left(\mathrm{p}^{\prime}\right) \cap \operatorname{intB}(x, \varepsilon) \neq \emptyset, \quad \forall \mathrm{p}^{\prime} \in \mathrm{B}(\mathrm{p}, \mathrm{t}) .
$$

Since $x \in \widetilde{G}_{\omega}(p)$, we have that $0 \in F(\omega, x, p)$ and $x \in \operatorname{int} B\left(x_{0}, r\right)$. Choose $0<\eta<\varepsilon$ such that $B(x, \eta) \subset$ $B\left(x_{0}, r\right)$ and $B(p, \eta) \subset B\left(p_{0}, r\right)$. Arguing as above for the pair $(x, p)$ in the place of $\left(x_{0}, p_{0}\right)$, the constant $\eta$ in the place of $r$, and the ball $B(x, \eta), B(0, \eta), B(p, \eta), B\left(0, \frac{\eta \sigma}{1+\sigma}\right)$ instead of $B\left(x_{0}, r\right), B(0, r), B\left(p_{0}, r\right), B\left(0, \frac{r \sigma}{1+\sigma}\right)$, respectively, we find a constant $0<t<\eta$ such that

$$
\mathrm{G}\left(\omega, \mathrm{p}^{\prime}\right) \cap \operatorname{int} \mathrm{B}(x, \eta) \neq \emptyset, \quad \forall \mathrm{p}^{\prime} \in \mathrm{B}(\mathrm{p}, \mathrm{t}) .
$$


Since $\operatorname{int} B(x, \eta) \subset \operatorname{int} B\left(x_{0}, r\right) \cap \operatorname{int} B(x, \varepsilon)$, from (3.4) we get

$$
\mathrm{G}\left(\omega, \mathrm{p}^{\prime}\right) \cap \operatorname{int} \mathrm{B}\left(x_{0}, \mathrm{r}\right) \cap \operatorname{int} \mathrm{B}(x, \varepsilon) \neq \emptyset, \quad \forall \mathrm{p}^{\prime} \in \mathrm{B}(\mathrm{p}, \mathrm{t}) .
$$

That is,

$$
\widetilde{\mathrm{G}}_{\omega}\left(\mathrm{p}^{\prime}\right) \cap \operatorname{intB}(x, \varepsilon) \neq \emptyset, \quad \forall p^{\prime} \in \mathrm{B}(\mathrm{p}, \mathrm{t})
$$

Remark 3.10. We notice that [17, Theorem 3.12] is required the assumption on inner semicompactness of the metric projection mapping, but Theorem 3.9 is not required. Moreover, [17, Theorem 3.12] uses normal coderivatives, while Theorem 3.9 uses Fréchet coderivatives. Also, we can see from the proof of Theorem 3.9 that the conclusion of the theorem is still valid, if the topological space P is replaced by a metric space.

When considering the deterministic case of Theorem 3.9, we have the following corollary.

Corollary 3.11. Suppose that all the assumptions of Corollary 3.3 are satisfied. Moreover, assume that

(iii) $)_{3}$ for any $(x, p) \in \mathrm{B}\left(x_{0}, r\right) \times \mathrm{B}\left(\mathrm{p}_{0}, \mathrm{r}\right)$, the multifunction $\mathrm{F}(x, \cdot)$ is l.s.c. at $\mathrm{p}$.

Then there exists a constant $\mathrm{s} \in(0, \mathrm{r})$ such that the multifunction $\widetilde{\mathrm{G}}: \mathrm{P} \rightrightarrows \mathrm{X}$ defined by

$$
\widetilde{G}(p):=G(p) \cap \operatorname{int} B\left(x_{0}, r\right),
$$

is nonempty and l.s.c. on $\mathrm{B}\left(\mathrm{p}_{0}, \mathrm{~s}\right)$.

Remark 3.12. Corollary 3.11 shows that the condition " $F\left(x_{0}, \cdot\right)$ is inner semicontinuous at $\left(\mathrm{p}_{0}, 0\right)$ " in $[2$, Corollary 3.3] and [3, Theorem 5.1] can be omitted. Moreover, Corollary 3.11 includes [8, Theorem 3.1] as a special case. We elaborate from the following four aspects:

(a) the condition " $F$ is nonempty-valued around $\left(x_{0}, p_{0}\right)$ " in [8, Theorem 3.1] is omitted;

(b) the condition "for each $p \in P$, the multifunction $F_{p}(\cdot)$ is closed-graph" in [8, Theorem 3.1] is replaced by the weaker condition "for each $p \in B\left(p_{0}, r\right)$, the multifunction $\mathrm{F}_{p}(\cdot)$ is closed" in Corollary 3.11;

(c) the condition (A1) in [8, Theorem 3.1] obviously implies the condition (ii) in Corollary 3.11;

(d) the condition (A2) in [8, Theorem 3.1] is omitted.

Example 3.13. Let $\mathrm{X}=\mathrm{Y}=\mathrm{P}=\mathbb{R}$. Then $\mathrm{X}$ and $\mathrm{Y}$ are separable Asplund spaces and $\mathrm{P}$ is a topological space. Moreover, let $\Omega=\{0,1\}, \mathcal{A}=\{\emptyset,\{0\},\{1\},\{0,1\}\}$. Then $(\Omega, \mathcal{A})$ is a measurable space. Define the function $\mu: \mathcal{A} \rightarrow \mathbb{R}$ as follows

$$
\mu(\emptyset)=0, \quad \mu(\{0\})=\mu(\{1\})=\frac{1}{2}, \quad \mu(\{0,1\})=1 .
$$

Obviously, $(\Omega, \mathcal{A}, \mu)$ is a complete $\sigma$-finite measure space. For any $(\omega, x, p) \in \Omega \times X \times P$, define the multifunction $\mathrm{F}: \Omega \times \mathrm{X} \times \mathrm{P} \rightrightarrows \mathrm{Y}$ as follows

$$
F(\omega, x, p)= \begin{cases}x-p+2, & \text { if } \omega=0 \\ x-\sqrt{p}, & \text { if } \omega=1\end{cases}
$$

Then for any $p \in P, F(\cdot, \cdot, p): \Omega \times X \rightrightarrows Y$ is measurable. In fact, for any closed subset $B \subset Y$, we have

$$
\begin{aligned}
\mathrm{F}_{\mathrm{p}}^{-1}(\mathrm{~B}) & =\{(\omega, x) \in \Omega \times X: F(\omega, x, p) \cap B \neq \emptyset\} \\
& =\{(0, x) \in \Omega \times X:\{x-p+2\} \cap B \neq \emptyset\} \cup\{(1, x) \in \Omega \times X:\{x-\sqrt{p}\} \cap B \neq \emptyset\} \\
& =\{0\} \times\{x \in X: x \in p-2+B\} \cup\{1\} \times\{x \in X: x \in \sqrt{p}+B\} \\
& =\{0\} \times(p-2+B) \cup\{1\} \times(\sqrt{p}+B) .
\end{aligned}
$$


Noting that a singleton set in $\mathbb{R}$ is closed, we obtain that $F_{p}^{-1}(B)$ is a closed subset of $\Omega \times X$. It follows that $\mathrm{F}(\cdot, \cdot, \mathrm{p}): \Omega \times \mathrm{X} \rightrightarrows \mathrm{Y}$ is measurable.

Furthermore, let $x_{0}=2, p_{0}=4$. Obviously, for all $\omega \in \Omega, 0 \in F\left(\omega, x_{0}, p_{0}\right)$. For any $\omega \in \Omega$, let $r=1$, $\sigma=1$. Then we have the following conclusions:

(i) For any $p \in \mathbb{R}, F_{\omega, p}(\cdot)$ is a continuous single-valued function. Hence, it has closed graph.

(ii) Since for all $(\omega, p) \in \Omega \times P, F_{\omega, p}(\cdot)$ is Fréchet differentiable, we obtain that $\nabla F_{\omega, p}(x)=I_{X}$, for all $x \in X$. By Lemma 2.7,

$$
\widehat{D}^{*} F_{\omega, p}(x, y)\left(y^{*}\right)=\left\{\nabla F_{\omega, p}(x)^{*} y^{*}\right\}=\left\{y^{*}\right\},
$$

and it follows that

$$
1 \leqslant \inf \left\{\left\|x^{*}\right\|: x^{*} \in \widehat{D}^{*} F_{\omega, p}(x, y)\left(y^{*}\right), \quad y \in B(0, r) \cap F_{\omega, p}(x),\left\|y^{*}\right\|=1\right\} .
$$

(iii) By the definition of $F$, for any $\omega \in \Omega, F(\omega, \cdot, \cdot)$ is continuous at $(x, p)$. It follows that $F(\omega, \cdot, \cdot)$ is 1.s.c. at $(2,4)$.

(iv) If $\omega=0$, let $\mathrm{V}=\mathrm{B}\left(\mathrm{x}_{0}, 1\right)=[1,3], \mathrm{U}=\mathrm{B}\left(\mathrm{p}_{0}, 1\right)=[3,5], \mathrm{l}=1$. Then we have

$$
x-p^{\prime}+2 \in x-p+2+\left|p^{\prime}-p\right| B_{Y}, \quad \forall x \in[1,3], \quad p, p^{\prime} \in[3,5] .
$$

That is,

$$
F\left(\omega, x, p^{\prime}\right) \subset F(\omega, x, p)+l \cdot\left|p^{\prime}-p\right| B_{Y}, \quad \forall x \in[1,3], \quad p, p^{\prime} \in[3,5] .
$$

If $\omega=1$, let $\mathrm{V}=\mathrm{B}\left(\mathrm{x}_{0}, 1\right)=[1,3], \mathrm{U}=\mathrm{B}\left(\mathrm{p}_{0}, 1\right)=[3,5], \mathrm{l}=2$. For any $\mathrm{p}, \mathrm{p}^{\prime} \in[3,5]$, we have $\left|\sqrt{\mathrm{p}}-\sqrt{\mathrm{p}^{\prime}}\right| \leqslant 3-1=2$, and it follows that

$$
x-\sqrt{p^{\prime}} \in x-\sqrt{p}+2\left|p^{\prime}-p\right| B_{Y}, \quad \forall x \in[1,3], \quad p, p^{\prime} \in[3,5] .
$$

That is,

$$
F\left(\omega, x, p^{\prime}\right) \subset F(\omega, x, p)+l \cdot\left|p^{\prime}-p\right| B_{Y}, \quad \forall x \in[1,3], p, p^{\prime} \in[3,5] .
$$

(v) By the definition of $F$, for any $(\omega, x, p) \in\{0,1\} \times \mathbb{R} \times \mathbb{R}, F(\omega, x, \cdot)$ is continuous at $p$. It follows that $F(\omega, x, \cdot)$ is 1.s.c. at $p$.

By the above discussions, we know that all the conditions of Theorems 3.1, 3.5, 3.7 and 3.9 are satisfied.

It follows that

(1) for any $p \in \mathbb{R}, \mathrm{G}(\cdot, \mathrm{p}):\{0,1\} \rightrightarrows \mathbb{R}$ is measurable;

(2) for any $\omega \in\{0,1\}, G(\omega, \cdot): \mathbb{R} \rightrightarrows \mathbb{R}$ is locally metrically regular around $(2,4)$ with modulus 2 . In fact, for any $\mu \in\left(0, \frac{1}{4}\right)$, we have

$$
\operatorname{dist}(x, G(\omega, p)) \leqslant 2 \operatorname{dist}(0, F(\omega, x, p)),
$$

for all $(x, p) \in B\left(2, \frac{1}{2}\right) \times B(4,1)$ with $\operatorname{dist}(0, F(\omega, x, p))<\mu$;

(3) for any $\omega \in\{0,1\}, G(\omega, \cdot): \mathbb{R} \rightrightarrows \mathbb{R}$ is metrically regular around $(2,4)$ with modulus 2;

(4) for any $\omega \in\{0,1\}, G(\omega, \cdot): \mathbb{R} \rightrightarrows \mathbb{R}$ is Lipschitz-like around $(2,4)$;

(5) for any $\omega \in\{0,1\}$, there exists a constant $s \in(0,1)$ such that the function $\widetilde{\mathrm{G}}_{\omega}: \mathbb{R} \rightrightarrows \mathbb{R}$ defined by

$$
\widetilde{G}_{\omega}(p):=G(\omega, p) \cap \operatorname{intB}(2,1),
$$

is nonempty and l.s.c. on $\mathrm{B}(4, \mathrm{~s})$. 


\section{Applications to random parametric generalized equations}

In this section, we consider a special case of $F$ in (1.4) which has the following form

$$
F(\omega, x, p)=f(\omega, x, p)+Q(\omega, x, p),
$$

where $\mathrm{f}: \Omega \times \mathrm{X} \times \mathrm{P} \rightarrow \mathrm{Y}$ is a single-valued mapping and $\mathrm{Q}: \Omega \times \mathrm{X} \times \mathrm{P} \rightrightarrows \mathrm{Y}$ is a multifunction. In this case, (1.4) becomes

$$
0 \in f(\omega, x, p)+Q(\omega, x, p) .
$$

The deterministic case of this random generalized equation was introduced by Robinson [13]. It is wellknown that the deterministic case of model (4.1) provides a convenient framework for the unified study of optimal solutions in many optimization-related areas including mathematical programming, complementarity, variational inequalities, optimal control, mathematical economics, equilibrium and some other fields (see, for example, $[9,10,16]$ and the references therein).

The solution map $\mathrm{G}: \Omega \times \mathrm{P} \rightrightarrows \mathrm{X}$ associated with (4.1) is defined by

$$
G(\omega, p):=\{x \in X: 0 \in f(\omega, x, p)+Q(\omega, x, p)\} .
$$

In what follows, we establish sufficient conditions ensuring the (local) metric regularity, the Lipschitz-like property, the nonemptiness and the lower semicontinuity of the solution map in (4.2).

Lemma 4.1 ([17, Lemma 4.3]). Let $(\Omega, \mathcal{A})$ be a measurable space, $\mathrm{X}$ a separable Banach space, $\mathrm{f}_{1}: \Omega \rightarrow \mathrm{X} a$ measurable mapping and $\mathrm{F}_{2}: \Omega \rightrightarrows \mathrm{X}$ a weakly measurable multifunction with closed values. Then $\mathrm{f}_{1}+\mathrm{F}_{2}: \Omega \rightrightarrows \mathrm{X}$ is weakly measurable.

Theorem 4.2. Let $\mathrm{X}$ be separable Asplund space, $\mathrm{Y}$ be $\sigma$-compact separable Asplund space, $\mathrm{P}$ a topological space, $(\Omega, \mathcal{A}, \mu)$ a complete $\sigma$-finite measure space, $\mathrm{f}: \Omega \times \mathrm{X} \times \mathrm{P} \rightarrow \mathrm{Y}$ a single-valued mapping and $\mathrm{Q}: \Omega \times \mathrm{X} \times$ $\mathrm{P} \rightrightarrows \mathrm{Y}$ a multifunction such that for each $\mathrm{p} \in \mathrm{P}, \mathrm{f}(\cdot, \cdot, \mathrm{p})$ is measurable and $\mathrm{Q}(\cdot, \cdot, \mathrm{p})$ is weakly measurable with closed values. Let $\mathrm{G}: \Omega \times \mathrm{P} \rightrightarrows \mathrm{X}$ the solution map defined by (4.2), and $\left(\mathrm{x}_{0}, \mathrm{p}_{0}\right) \in \mathrm{X} \times \mathrm{P}$ a pair such that $\left(\omega, x_{0}, p_{0},-f\left(\omega, x_{0}, p_{0}\right)\right) \in$ gph $Q$ for all $\omega \in \Omega$. Let $f_{\omega, p}(\cdot):=f(\omega, \cdot, p)$ and $Q_{\omega, p}(\cdot):=Q(\omega, \cdot, p)$. Suppose that for each $\omega \in \Omega$, there exist constants $r>0$ and $\sigma>0$ such that:

(i) for any $\mathrm{p} \in \mathrm{B}\left(\mathrm{p}_{0}, \mathrm{r}\right)$, the mapping $\mathrm{f}_{\omega, \mathrm{p}}(\cdot)$ is Fréchet differentiable on $\mathrm{X}$ and the multifunction $\mathrm{Q}_{\omega, \mathrm{p}}(\cdot)$ is closed;

(ii) for any $(x, p) \in B\left(x_{0}, r\right) \times B\left(p_{0}, r\right)$,

$$
\begin{gathered}
\sigma \leqslant \inf \left\{\left\|x^{*}\right\|: x^{*} \in \nabla f_{\omega, p}(x)^{*} y^{*}+\widehat{D}^{*} Q_{\omega, p}\left(x, y-f_{\omega, p}(x)\right)\left(y^{*}\right),\right. \\
\left.y \in B(0, r) \cap\left(f_{\omega, p}(x)+Q_{\omega, p}(x)\right),\left\|y^{*}\right\|=1\right\} .
\end{gathered}
$$

Then

(1) for any $\mathrm{p} \in \mathrm{P}, \mathrm{G}(\cdot, \mathrm{p}): \Omega \rightrightarrows \mathrm{X}$ is measurable;

(2) for any $\omega \in \Omega, \mathrm{G}(\omega, \cdot): \mathrm{P} \rightrightarrows \mathrm{X}$ is locally metrically regular around $\left(\mathrm{x}_{0}, \mathrm{p}_{0}\right)$ with modulus $\frac{1+\sigma}{\sigma}$. In fact, for any $\mu \in\left(0, \frac{r \sigma}{2(1+\sigma)}\right)$, we have

$$
\operatorname{dist}(\chi, \mathrm{G}(\omega, \mathrm{p})) \leqslant \frac{1+\sigma}{\sigma} \operatorname{dist}(-\mathrm{f}(\omega, \chi, \mathrm{p}), \mathrm{Q}(\omega, \chi, \mathrm{p})),
$$

for all $(x, p) \in B\left(x_{0}, \frac{r}{2}\right) \times B\left(p_{0}, r\right)$ with $\operatorname{dist}(-f(\omega, x, p), Q(\omega, x, p))<\mu$.

Proof. Define the multifunction $\mathrm{F}: \Omega \times \mathrm{X} \times \mathrm{P} \rightrightarrows \mathrm{Y}$ by

$$
F(\omega, x, p)=f(\omega, x, p)+Q(\omega, x, p), \quad \forall(\omega, x, p) \in \Omega \times X \times P .
$$

Let $F_{\omega, p}(\cdot):=F(\omega, \cdot p)$. Then $F_{\omega, p}(\cdot)=f_{\omega, p}(\cdot)+Q_{\omega, p}(\cdot)$. Obviously, $\left(\omega, x_{0}, p_{0},-f\left(\omega, x_{0}, p_{0}\right)\right) \in$ gph Q 
is equivalent to $0 \in \mathrm{F}\left(\omega, x_{0}, p_{0}\right)$. It follows from Lemma 4.1 that for each $p \in P, F(\cdot, \cdot, p): \Omega \times X \rightrightarrows Y$ is weakly measurable. Since $Y$ is $\sigma$-compact, by [4, Theorem 3.2 (ii)], we have that $F(\cdot, \cdot, p): \Omega \times X \rightrightarrows Y$ is measurable. Fix any $\omega \in \Omega$, there exist constants $r>0$ and $\sigma>0$ such that conditions (i) and (ii) are satisfied. By condition (i), it is easy to check that for any $p \in B\left(p_{0}, r\right)$, the multifunction $F_{\omega, p}(\cdot)$ is closed. By condition (ii) and Lemma 2.8, we obtain that for any $(x, p) \in B\left(x_{0}, r\right) \times B\left(p_{0}, r\right)$,

$$
\sigma \leqslant \inf \left\{\left\|x^{*}\right\|: x^{*} \in \widehat{D}^{*} F_{\omega, p}(x, y)\left(y^{*}\right), y \in B(0, r) \cap F_{\omega, p}(x),\left\|y^{*}\right\|=1\right\} .
$$

Therefore, Theorem 4.2 follows from Theorem 3.1.

Remark 4.3. Theorem 4.2 is similar to [17, Theorem 4.4 ]. In Theorem 4.2, the assumption "for any $p \in$ $B\left(p_{0}, r\right)$, the mapping $f_{\omega, p}(\cdot)$ is Fréchet differentiable on $X^{\prime \prime}$ is required, while in [17, Theorem 4.4 ], the assumption "for any $p \in B\left(p_{0}, r\right)$, the mapping $f_{\omega, p}(\cdot)$ is strictly differentiable on $X$ " is required.

Theorem 4.4. Suppose that all the assumptions of Theorem 4.2 are satisfied. Moreover, assume that

(iii) $)_{1}$ for any $\omega \in \Omega, f(\omega, \cdot, \cdot)$ is continuous at $\left(x_{0}, \mathrm{p}_{0}\right)$ and $\mathrm{Q}(\omega, \cdot, \cdot)$ is l.s.c at $\left(\mathrm{x}_{0}, \mathrm{p}_{0}\right)$.

Then

(1) for any $\mathrm{p} \in \mathrm{P}, \mathrm{G}(\cdot, \mathrm{p}): \Omega \rightrightarrows \mathrm{X}$ is measurable;

(2) for any $\omega \in \Omega, G(\omega, \cdot): P \rightrightarrows X$ is metrically regular around $\left(x_{0}, p_{0}\right)$ with modulus $\frac{1+\sigma}{\sigma}$.

Theorem 4.5. Suppose that all the assumptions of Theorem 4.2 are satisfied. Moreover, assume that $\mathrm{P}$ is a subset of a normed space and

(iii) $)_{2}$ for any $\omega \in \Omega$, there exist a neighborhood $\mathrm{V}$ of $\mathrm{x}_{0}$, a neighborhood $\mathrm{U}$ of $\mathrm{p}_{0}$ and a constant $\mathrm{l}>0$ such that

$$
f\left(\omega, x, p^{\prime}\right)+Q\left(\omega, x, p^{\prime}\right) \subset f(\omega, x, p)+Q(\omega, x, p)+l\left\|p^{\prime}-p\right\| B_{Y}, \quad \forall x \in V, \quad \forall p, p^{\prime} \in U
$$

Then

(1) for any $\mathrm{p} \in \mathrm{P}, \mathrm{G}(\cdot, \mathrm{p}): \Omega \rightrightarrows \mathrm{X}$ is measurable;

(2) for any $\omega \in \Omega, \mathrm{G}(\omega, \cdot): \mathrm{P} \rightrightarrows \mathrm{X}$ is Lipschitz-like around $\left(\mathrm{p}_{0}, \mathrm{x}_{0}\right)$.

Theorem 4.6. Suppose that all the assumptions of Theorem 4.2 are satisfied. Moreover, assume that

(iii) $)_{3}$ for any $(\omega, x, p) \in \Omega \times \mathrm{B}\left(x_{0}, r\right) \times \mathrm{B}\left(\mathrm{p}_{0}, \mathrm{r}\right)$, the mapping $\mathrm{f}(\omega, \mathrm{x}, \cdot)$ is continuous at $\mathrm{p}$ and the multifunction $\mathrm{Q}(\omega, x, \cdot)$ is l.s.c. at $\mathrm{p}$.

Then

(1) for any $\mathrm{p} \in \mathrm{P}, \mathrm{G}(\cdot, \mathrm{p}): \Omega \rightrightarrows \mathrm{X}$ is measurable;

(2) for any $\omega \in \Omega$, there exists a constant $\mathrm{s} \in(0, \mathrm{r})$ such that $\widetilde{\mathrm{G}}_{\omega}: \mathrm{P} \rightrightarrows \mathrm{X}$ defined by

$$
\widetilde{\mathrm{G}}_{\omega}(\mathrm{p}):=\mathrm{G}(\omega, \mathrm{p}) \cap \operatorname{int} \mathrm{B}\left(\mathrm{x}_{0}, \mathrm{r}\right),
$$

is nonempty and l.s.c. on $\mathrm{B}\left(\mathrm{p}_{0}, \mathrm{~s}\right)$.

Remark 4.7. We notice that [17, Theorem 4.4, Theorem 4.7-4.9] are always required the assumption on inner semicompactness of the metric projection mapping, but Theorems 4.2, 4.4, 4.5 and 4.6 are not required. Moreover, [17, Theorem 4.4, Theorem 4.7-4.9] use normal coderivatives, while Theorems 4.2, 4.4, 4.5 and 4.6 use Fréchet coderivatives. 


\section{Acknowledgment}

The work is supported by the National Natural Science Foundation of China (No.71372113, 71531005, 11226228) and China Postdoctoral Science Foundation (No.2014M551312). The authors are grateful to the editor and referees for their valuable comments and suggestions.

\section{References}

[1] J. P. Aubin, H. Frankowska, Set-valued analysis, Systems \& Control: Foundations \& Applications, Birkhäuser Boston, Inc., Boston, MA, (1990). 2, 2.10

[2] M. Durea, Openness properties for parametric set-valued mappings and implicit multifunctions, Nonlinear Anal., 72 (2010), 571-579. 1, 1, 3.12

[3] M. Durea, R. Strugariu, Quantitative results on openness of set-valued mappings and implicit multifunction theorems, Pac. J. Optim., 6 (2010), 533-549. 1, 1, 3.12

[4] C. J. Himmelberg, Measurable relations, Fund. Math., 87 (1975), 53-72. 2, 2.9, 4

[5] N. Q. Huy, D. S. Kim, K. V. Ninh, Stability of implicit multifunctions in Banach spaces, J. Optim. Theory Appl., 155 (2012), 558-571. 1, 1, 3.4

[6] N. Q. Huy, J.-C. Yao, Stability of implicit multifunctions in Asplund spaces, Taiwanese J. Math., 13 (2009), 47-65. 1, 1, 3.4

[7] Y. S. Ledyaev, Q. J. Zhu, Implicit multifunction theorems, Set-Valued Anal., 7 (1999), 209-238. 1

[8] G. M. Lee, N. N. Tam, N. D. Yen, Normal coderivative for multifunctions and implicit function theorems, J. Math. Anal. Appl., 338 (2008), 11-22. 1, 1, 3.12

[9] B. S. Mordukhovich, Variational analysis and generalized differentiation, I, Basic theory, Grundlehren der Mathematischen Wissenschaften [Fundamental Principles of Mathematical Sciences], Springer-Verlag, Berlin, (2006). 2, 2.1, $2.2,2.3,2.4,2.5,2,2.6,2.7,2.8,4$

[10] B. S. Mordukhovich, Variational analysis and generalized differentiation, II, Applications, Grundlehren der Mathematischen Wissenschaften [Fundamental Principles of Mathematical Sciences], Springer-Verlag, Berlin, (2006). 2, 4

[11] H. V. Ngai, M. Théra, Error bounds and implicit multifunction theorem in smooth Banach spaces and applications to optimization, Set-Valued Anal., 12 (2004), 195-223. 1

[12] S. M. Robinson, Stability theory for systems of inequalities, I, Linear systems, SIAM J. Numer. Anal., 12 (1975), 754-769. 1

[13] S. M. Robinson, Stability theory for systems of inequalities, II, Differentiable nonlinear systems, SIAM J. Numer. Anal., 13 (1976), 497-513. 4

[14] S. M. Robinson, Generalized equations and their solutions, I, Basic theory, Point-to-set maps and mathematical programming, Math. Programming Stud., 10 (1979), 128-141.

[15] S. M. Robinson, Generalized equations and their solutions, II, Applications to nonlinear programming, Optimality and stability in mathematical programming, Math. Programming Stud., 19 (1982), 200-221. 1

[16] R. T. Rockafellar, R. J.-B. Wets, Variational analysis, Grundlehren der Mathematischen Wissenschaften [Fundamental Principles of Mathematical Sciences], Springer-Verlag, Berlin, (1998). 4

[17] M.-G. Yang, N.-J. Huang, Random implicit function theorems in Asplund spaces with applications, J. Nonlinear Convex Anal., 14 (2013), 497-517. 1, 1, 3.2, 3.6, 3.8, 3.10, 4.1, 4.3, 4.7

[18] M.-G. Yang, Y.-F. Xu, Openness results for parametric set-valued mappings in Asplund spaces, Optim. Lett., 8 (2014), 2227-2243. 1

[19] N. D. Yen, Implicit function theorems for set-valued maps, Acta Math. Vietnam, 12 (1987), 17-28. 1 